\title{
Asociación entre el tiempo de inicio de la ventilación no invasiva y la mortalidad en el edema pulmonar cardiogénico
}

\author{
Relationship between timing of initiation of non-invasive ventilation and mortality in \\ cardiogenic pulmonary edema
}

\author{
Santiago Orozco-Montoya ${ }^{1 *}$, Gustavo A. Crespo-Habib ${ }^{1}$, María Eugenia Peña-Montoya ${ }^{1,2}$, \\ Fabián A. Jaimes-Barragán ${ }^{1}$ y Andrés Zapata-Cárdenas ${ }^{1,3}$; en nombre del Grupo de Investigación en \\ Medicina Interna (GIMI), Facultad de Medicina, Universidad de Antioquia \\ ${ }^{1}$ Facultad de Medicina, Universidad de Antioquia; ${ }^{2}$ Clínica SOMA; ${ }^{3}$ Unidad Funcional de Enfermedades Crónicas del Adulto, Hospital Universitario \\ San Vicente Fundación. Medellín, Colombia
}

\section{Resumen}

Introducción: La ventilación no invasiva reduce la necesidad de intubación y la estancia en la unidad de cuidados intensivos en los pacientes con edema pulmonar cardiogénico. Objetivo: Evaluar la posible asociación entre el inicio de la ventilación no invasiva desde el ingreso a urgencias con la mortalidad y el requerimiento de intubación en pacientes con edema pulmonar cardiogénico. Método: Estudio analítico de cohorte retrospectiva, en el que se revisaron las historias clínicas de pacientes mayores de 18 años hospitalizados en una institución de Medellín, Colombia. Resultados: 70 pacientes cumplieron los criterios de inclusión y se compararon según el estado vital al alta. Sobrevivientes (49): la edad promedio fue de 63 años, el $34.7 \%$ fueron mujeres, el 57.1\% tuvieron historia de neumopatía crónica y el $89 \%$ eran hipertensos; el promedio para recibir la intervención fue de 10 horas y el 20\% requirieron intubación orotraqueal. No sobrevivientes (21): la edad promedio fue de 74 años, el 57.1\% fueron mujeres, el 57.1\% tuvieron historia de neumopatía crónica y el 90\% eran hipertensos; el promedio para recibir la intervención fue de 7 horas y el 62\% requirieron intubación. Tiempo de inicio en relación con la mortalidad en el análisis multivariado: odds ratio (OR) 1,05, intervalo de confianza del 95\% (IC95\%) 0.89-1.24, $p=0.499 ; y$ por variable instrumental: $7 \%$ de diferencia de medias. En cuanto al tiempo de inicio y su asociación con la necesidad de intubación: OR 0.93, IC95\% 0,86-1,01. Conclusiones: Este estudio sugiere que el inicio tardío de la ventilación no invasiva es un factor de riesgo; sin embargo, no se halló asociación estadísticamente significativa, por lo que se requieren estudios adicionales para confirmar este hallazgo.

Palabras clave: Ventilación no invasiva. Edema pulmonar. Insuficiencia cardíaca. Mortalidad.

\section{Abstract}

Introduction: Non-invasive ventilation reduces the need for intubation and intensive care stay in cardiogenic pulmonary edema. Objective: To evaluate the possible relationship between the initiation of non-invasive ventilation on admission to the emergency room and mortality and the need for intubation in patients with cardiogenic pulmonary edema. Method: $A$ retrospective analytical cohort study. A chart review of patients over the age of 18 hospitalized at an institution in Medellín, Colombia. Results: 70 patients met the inclusion criteria and were compared by vital status at discharge. Survivors (49):

\section{Correspondencia:}

*Santiago Orozco-Montoya

E-mail: santiorozmontoya @ hotmail.com
Disponible en internet: 22-02-2022 Rev Colomb Cardiol. 2022;29(1):41-48 www.rccardiologia.com 0120-5633 / C 2020 Sociedad Colombiana de Cardiología y Cirugía Cardiovascular. Publicado por Permanyer. Este es un artículo open access bajo la licencia CC BY-NC-ND (http://creativecommons.org/licenses/by-nc-nd/4.0/). 
the average age was 63 years, $34.7 \%$ were women, $57.1 \%$ had a history of chronic pulmonary disease, $89 \%$ had hypertension, 10 hours was the average for receiving the intervention, and $20 \%$ required orotracheal intubation. Non-survivors (21): the average age was 74 years, $57.1 \%$ were women, $57.1 \%$ had a history of chronic pulmonary disease, $90 \%$ had hypertension; 7 hours was the average for receiving the intervention, and $62 \%$ required intubation. Timing of initiation related to mortality on the multivariate analysis: odds ratio (OR) 1.05, 95\% confidence interval $(95 \% \mathrm{Cl}) 0.89-1.24, p=0.499$; and by instrumental variable: $7 \%$ difference in means. With regard to timing of initiation and its association with the need for intubation: OR $0.93,95 \% \mathrm{Cl}$ 0.86-1.01. Conclusions: This study suggests that late initiation of non-invasive ventilation is a risk factor; however, no statistically significant association was found. Therefore, further studies are needed to confirm this finding.

Keywords: Noninvasive ventilation. Pulmonary edema. Heart failure. Mortality.

\section{Introducción}

El edema pulmonar cardiogénico (EPC) es una condición que conlleva una significativa mortalidad intrahospitalaria, que en el caso del infarto agudo de miocardio puede ser hasta del $10 \%$, a pesar de su reconocimiento temprano y del gran número de medidas farmacológicas disponibles ${ }^{1-3}$. Una cantidad importante de pacientes requerirán medidas invasivas que garanticen la oxigenación, debido a las alteraciones en el intercambio gaseoso y en la dinámica respiratoria desencadenadas por esta condición; para esto, como alternativa se plantea el uso de ventilación mecánica no invasiva $(\mathrm{VMNI})^{4}$. Hay dos principales modalidades de VMNI: presión positiva continua de la vía aérea (CPAP) y ventilación con presión soporte (NIPSV o BPAP). Ambas modalidades parecen ser efectivas en los pacientes con EPC mediante la reducción del esfuerzo respiratorio $y$, por consiguiente, de las tasas de intubación orotraqueal, comparadas con la terapia convencional ${ }^{5,6}$.

Los anteriores resultados contrastan con los obtenidos en el ensayo clínico aleatorizado más grande en el tema, que fue publicado en el año 2008 por Gray et al. ${ }^{7}$ (3-CPO), el cual incluyó 1069 pacientes con EPC agudo y acidosis $(\mathrm{pH}<7.35)$. Los participantes fueron asignados a CPAP, NIPSV o terapia con oxígeno convencional, sin encontrar diferencias en cuanto a las tasas de intubación o la mortalidad en los días 7 y 30 , aunque ambas técnicas mejoraron la dificultad respiratoria en forma más rápida que la terapia convencional. Debido a las características de los pacientes (se excluyeron los más graves y que potencialmente podrían haberse beneficiado más de la intervención) y a que hasta un $20 \%$ de los pacientes no terminaron el tratamiento asignado, consideramos que estos datos no representan de manera fidedigna todo el cuerpo de evidencia en el tema ${ }^{7}$. Otros estudios observacionales y metaanálisis parecen sugerir evidencia a favor del uso de VMNI en este escenario, con una aparente reducción de la mortalidad y de la necesidad de intubación orotraqueal ${ }^{8-11}$.

La VMNI es una estrategia de tratamiento que no tiene aceptación unánime en cuanto al impacto en la mortalidad; sus beneficios sobre la mecánica respiratoria, sin embargo, sí son claros $2,3,12-14$. Por esto, la VMNI es una terapia de segunda línea y en la práctica clínica no hay claridad sobre el momento más adecuado para iniciarla ${ }^{2,3,15,16}$. Partiendo de que puede tener impacto en desenlaces de importancia clínica, el objetivo principal de este estudio fue evaluar la posible asociación entre el tiempo de inicio de la VMNI desde el ingreso al servicio de urgencias con la mortalidad y el requerimiento de intubación en pacientes hospitalizados por EPC.

\section{Método}

Estudio de cohorte retrospectiva, con fuente secundaria de información basada en el registro electrónico de historias clínicas.

Se tomaron todos los registros de los pacientes que consultaron al servicio de urgencias con EPC en el periodo entre enero de 2013 y abril de 2018. El Hospital Universitario San Vicente Fundación (Medellín, Colombia) es una institución de cuarto nivel de atención y centro de referencia en el Centro-Occidente del país, con 694 camas y 112.036 consultas anuales por urgencias $^{17}$.

Se hizo una búsqueda de aquellos pacientes en cuya historia clínica se hubiera registrado el diagnóstico de edema pulmonar agudo, insuficiencia respiratoria aguda 0 falla cardíaca (códigos CIE-10 J81X, J96, I50), y que hubieran sido admitidos a la institución en el periodo comprendido entre enero de 2013 y abril de 2018. Adicionalmente, con el objetivo de facilitar la búsqueda, estos diagnósticos se cruzaron con el uso de infusión de nitroglicerina 
(asumiendo que los vasodilatadores hacen parte del manejo de primera línea del EPC) o con el ingreso a la unidad de cuidados intensivos (UCl). De la lista final de pacientes, los criterios de selección fueron: mayores de 18 años con criterios clínicos (disnea, ortopnea, falla ventilatoria) e imagenológicos (signos de congestión en la radiografía de tórax $)^{4}$ de EPC y que recibieron VMNI en las primeras 24 horas de ingreso a la institución.

Por último, se buscó en el registro manual (en el que solo había datos de los años 2016-2017) qué pacientes recibieron VMNI bajo el diagnóstico de EPC, verificando que no se repitieran pacientes encontrados previamente en el sistema.

Finalmente, se revisaron las historias de aquellos pacientes con los códigos diagnósticos y que hubieran recibido nitroglicerina, VMNI en urgencias o que hubieran ingresado a la $\mathrm{UCl}$.

Se excluyeron los pacientes menores de 18 años, los que recibieron VMNI luego de las primeras 24 horas desde el ingreso (en quienes la intervención ya no modificaría la evolución natural de la enfermedad) o que fueron intubados desde el inicio de la atención, mujeres en embarazo, quienes tuvieron contraindicaciones para el uso de VMNI, como paro cardíaco o respiratorio, encefalopatía grave, hemorragia gastrointestinal grave, inestabilidad hemodinámica, cirugía o traumatismo facial, obstrucción de la vía respiratoria superior, alto riesgo de aspiración o incapacidad para proteger la vía respiratoria e incapacidad para eliminar secreciones. También se excluyeron aquellos en los que se documentó otra enfermedad pulmonar descompensada en forma concomitante, como embolia pulmonar, neumotórax, neumonía y otras infecciones respiratorias.

La variable de exposición principal fue el tiempo de inicio de la VMNI, tomando como tiempo 0 el momento de admisión al servicio de urgencias con diagnóstico de EPC, mientras que la variable de desenlace principal fue el estado vital del paciente al momento del alta. Como desenlace secundario se exploró la necesidad de intubación.

Se consideraron como variables de confusión ${ }^{18}$, de acuerdo con la literatura revisada ${ }^{19-24}$, la edad, el sexo femenino, la falla cardíaca aguda de novo, los episodios previos de descompensación, el antecedente de anemia, el antecedente de enfermedad pulmonar obstructiva crónica, un factor desencadenante reconocido, los días de hospitalización, el nitrógeno ureico en sangre y la hemoglobina. Adicionalmente, por su importancia clínica, se ingresaron al modelo la necesidad de intubación posterior a la VMNI y los días en la UCl.
La fuente de la información recolectada para la presente investigación fue la historia clínica electrónica de los pacientes.

Adicionalmente, con el objetivo de mejorar el control de la confusión y la confusión residual, así como el probable «sesgo de confusión por indicación» ${ }^{25}$, se realizó un análisis de variables instrumentales basado en el supuesto de la existencia de una variable que se asociara con la exposición de interés, en este caso el tiempo hasta el inicio de la VMNI, pero no se asoció con el desenlace de mortalidad hospitalaria ${ }^{26}$, por lo que se tomó como variable instrumental la hora de ingreso a la institución.

Las variables cuantitativas fueron analizadas bajo estadísticas descriptivas, tales como promedios y rangos intercuartílicos, y fueron ingresadas a los modelos como variables continuas.

\section{Métodos estadísticos}

Para el análisis univariado se describieron distribuciones de frecuencia y porcentuales, así como medias, medianas y medidas de dispersión para variables cuantitativas.

Para el análisis multivariado se realizó una regresión logística múltiple, con la mortalidad al alta hospitalaria como variable dependiente y como variables independientes la exposición principal en horas y las variables consideradas potenciales confusoras. Los resultados se presentan como razones de disparidad (OR, odds ratio) con sus respectivos intervalos de confianza del 95\% (IC95\%). Así mismo, se realizó una segunda regresión logística simple de carácter exploratorio, con la necesidad de intubación como variable dependiente y el tiempo de inicio de la VMNI como variable independiente.

Adicionalmente, para el análisis de variables instrumentales se midió la variable hora de ingreso a la institución como hora militar, y se utilizó el método de mínimos cuadrados en dos estados (2SLS) ${ }^{27}$.

Se incluyeron en el análisis solo aquellos pacientes con el $100 \%$ de los datos diligenciados, y la información fue analizada con la ayuda del paquete estadístico Stata 16 (Stata Statistical Software: Release 16. StataCorp LLC, College Station, Texas, USA).

\section{Resultados}

\section{Participantes}

La muestra inicial correspondió a 2562 individuos que tenían el diagnóstico de edema pulmonar agudo, 
insuficiencia respiratoria aguda o falla cardíaca entre los años 2013 y 2018, o que habían recibido VMNI en el servicio de urgencias. De estos, se seleccionaron 1517 pacientes ya que habían recibido nitroglicerina 0 ingresaron a la UCl. Luego de aplicar los criterios de inclusión y exclusión quedaron 70 pacientes para el análisis final (Fig. 1).

\section{Datos descriptivos}

Las características clínicas más relevantes y los desencadenantes más frecuentes de EPC, según el estado vital al alta, se observan en la tabla 1.

Las características de la intervención, los datos de laboratorio al ingreso y los desenlaces principales, según el estado vital al alta, pueden encontrarse en la tabla 2.

\section{Resultados}

El tiempo de inicio de la VMNI (variable independiente), medido en horas cumplidas desde el ingreso al servicio de urgencias, en relación con la mortalidad intrahospitalaria (variable dependiente), luego del análisis entre estas dos variables arrojó una OR de 0.93 (IC95\%: 0.85-1.014). Luego del ajuste por variables de confusión se encontró que cada hora de retraso en el inició de la VMNI se asoció con un incremento del $5 \%$ en la mortalidad (OR: 1.05; IC95\%: 0.89-1.24), siendo no significativa estadísticamente. También se encontraron otras variables que pueden asociarse con mayor mortalidad, aunque no significativamente: la edad, un primer evento de falla cardíaca, la historia de episodios de descompensación de falla cardíaca, el tiempo de estancia en la UCl y la necesidad de intubación luego de haber recibido VMNI. Por otro lado, sugieren ser elementos protectores para mortalidad, pero sin asociación significativa, el sexo femenino, el antecedente de anemia, la historia de neumopatía crónica, el reconocimiento del desencadenante del EPC, los días de hospitalización totales y los valores del nitrógeno ureico y de la hemoglobina mayores al ingreso (Tabla 3).

\section{Otros análisis}

En cuanto al tiempo de inicio de la VMNI y su asociación con la necesidad de intubación endotraqueal, no se halló una asociación clara (OR: 0.93; IC95\%: 0.86-1.01).

El análisis con la variable instrumental de hora de ingreso al hospital sugiere un aumento de un $7 \%$ en el riesgo absoluto de morir por cada hora de diferencia

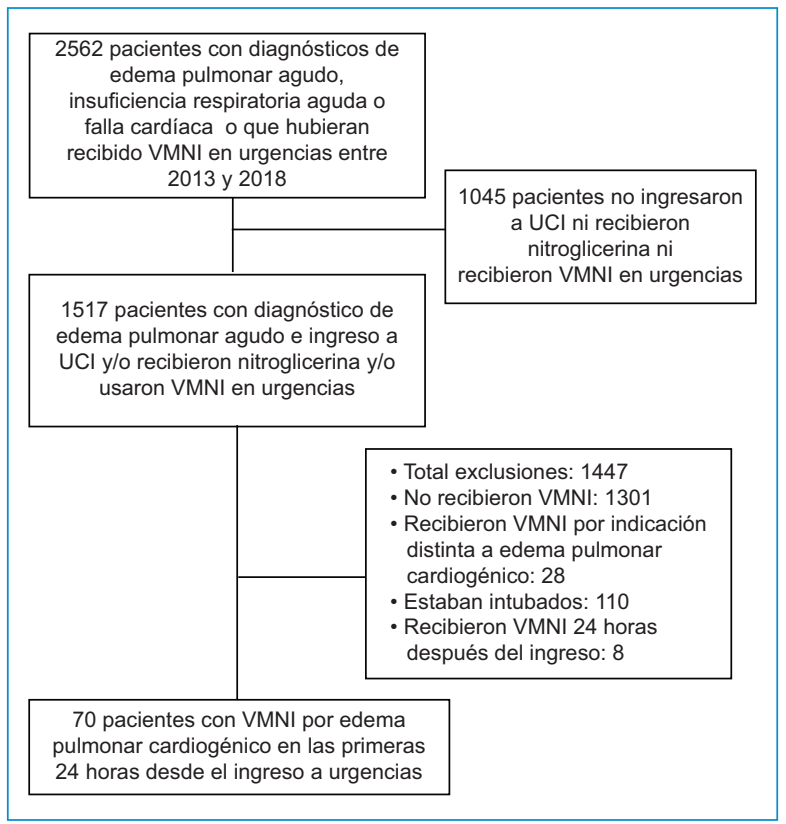

Figura 1. Selección de los pacientes. UCl: unidad de cuidados intensivos; VMNI: ventilación mecánica no invasiva.

en el inicio de la VMNI (OR: 0.07; IC95\%: $-0.44-0.56)$.

\section{Discusión}

El efecto biológico de la VMNI consiste en la reducción del esfuerzo respiratorio y sus efectos hemodinámicos en los pacientes con EPC ${ }^{4,12,9}$. En las guías colombianas y de la Sociedad Europea de Cardiología (SEC) sobre el manejo de la falla cardíaca ${ }^{2,14}$, la recomendación de VMNI en los pacientes con EPC es para la mejoría de la disnea y la acidemia que no respondan a las medidas del manejo convencional, y desestimulan el uso rutinario de VMNI en el EPC, ya que no encontraron impacto en la mortalidad, aunque la guía de la SEC sugiere que puede reducir la necesidad de intubación y las tasas de mortalidad (recomendación de clase lla: hay evidencia conflictiva o divergencia de la opinión, pero el peso de la opinión está a favor de su eficacia). La otra tendencia en la literatura está representada por las recomendaciones de las guías NICE (National Institute for Health and Care Excellence), la Sociedad Europea de Urgencias y algunos metaanálisis ${ }^{3,10,28,29}$ que recomiendan el uso de VMNI por sus efectos sobre la reducción de la mortalidad. Por la discordancia entre unas y otras fuentes, un metaanálisis de la Cochrane de $2019^{29}$ se propuso 
Tabla 1. Características de los pacientes según su estado vital al alta

\begin{tabular}{|c|c|c|}
\hline Características & $\begin{array}{l}\text { Sobrevivientes } \\
\qquad(\mathrm{n}=49)^{*}\end{array}$ & $\begin{array}{l}\text { No sobrevivientes } \\
\qquad(\mathrm{n}=21)^{*}\end{array}$ \\
\hline Edad, años & $63(22-87)$ & $74(40-84)$ \\
\hline Sexo masculino & $17(34.7)$ & $9(42.9)$ \\
\hline Sexo femenino & $32(65.3)$ & $12(57.1)$ \\
\hline $\begin{array}{l}\text { Antecedentes } \\
\text { Anemia } \\
\text { Fibrilación auricular } \\
\text { EPOC } \\
\text { Enfermedad coronaria } \\
\text { ECV } \\
\text { Diabetes mellitus } \\
\text { Hipertensión arterial }\end{array}$ & $\begin{array}{c}12(24.5) \\
6(12.2) \\
28(57.1) \\
8(16.3) \\
2(4.1) \\
17(34.7) \\
43(89)\end{array}$ & $\begin{array}{c}4(19) \\
2(9.5) \\
12(57.1) \\
8(38.1) \\
1(4.8) \\
6(28.6) \\
19(90)\end{array}$ \\
\hline $\begin{array}{l}\text { Tiempo de falla } \\
\text { cardíaca } \\
\text { Crónica } \\
\text { De novo }\end{array}$ & $\begin{array}{l}16(32.7) \\
33(67.3)\end{array}$ & $\begin{array}{l}6(28.6) \\
15(71.4)\end{array}$ \\
\hline $\begin{array}{l}\text { Factor desencadenante } \\
\text { Isquemia } \\
\text { Hipertensión arterial } \\
\text { Anemia } \\
\text { Infección } \\
\text { Nefropatía } \\
\text { TSV } \\
\text { No adherencia al } \\
\text { tratamiento } \\
\text { Insuficiencia aórtica }\end{array}$ & $\begin{array}{c}16(33) \\
13(26) \\
1(2) \\
12(24.5) \\
5(10) \\
1(2) \\
1(2) \\
0\end{array}$ & $\begin{array}{c}7(33) \\
1(4.8) \\
1(4.8) \\
7(33) \\
3(14.3) \\
1(4.8) \\
0 \\
1(4.8)\end{array}$ \\
\hline
\end{tabular}

resolver esta polémica y comparte 16 de sus 24 estudios con las fuentes de literatura previas (incluyendo 3-CPO y otros nueve ensayos clínicos de alta calidad), dando como resultado que, a pesar de que la evidencia no es de alta calidad (predominan los sesgos en cegamiento, reporte y seguimiento), la VMNI sí reduciría la mortalidad en los pacientes con EPC, incluyendo el ámbito de urgencias y de $\mathrm{UCl}$. Por lo anterior, consideramos que la evidencia más reciente sugiere un impacto de la VMNI sobre la mortalidad, lo cual justifica estudiar factores que modifiquen esta asociación, como lo es la temporalidad.

Nuestro trabajo es el primero en estudiar la relación entre el tiempo de inicio de la VMNI desde el ingreso al servicio de urgencias y la mortalidad. No encontramos ningún estudio que haya abordado el impacto del retraso en la VMNI, y las guías de práctica clínica contienen recomendaciones dispares sobre el momento adecuado de iniciarla ${ }^{2,3,13,15}$. Aún es poca la literatura en la que se
Tabla 2. Intervenciones y desenlaces según el estado vital al alta

\begin{tabular}{|c|c|c|}
\hline Intervención & $\begin{array}{l}\text { Sobrevivientes } \\
\qquad(\mathrm{n}=49)^{*}\end{array}$ & $\begin{array}{l}\text { No sobrevivientes } \\
\qquad(n=21)^{*}\end{array}$ \\
\hline $\begin{array}{l}\text { Tiempo de inicio de } \\
\text { VMNI, horas }\end{array}$ & $10(1-24)$ & $7(1-23)$ \\
\hline Recibió diurético & $41(84)$ & $19(90)$ \\
\hline Dosis de diurético, mg & $51(10-150)$ & $52(10-160)$ \\
\hline $\begin{array}{l}\text { Datos al ingreso } \\
\text { Hemoglobina, g/dl } \\
\text { BUN, mg/dl } \\
\text { Fracción de eyección, \% }\end{array}$ & $\begin{array}{c}13(8-17) \\
33(6-93) \\
53(14-80)\end{array}$ & $\begin{array}{c}13(7-17) \\
27(10-69) \\
50(10-83)\end{array}$ \\
\hline Disfunción diastólica & $42(85)$ & $18(85)$ \\
\hline $\begin{array}{l}\text { Desenlaces } \\
\text { TOT } \\
\text { Tiempo de estancia } \\
\text { en UCl, días } \\
\text { Tiempo de estancia } \\
\text { hospitalaria total, días }\end{array}$ & $\begin{array}{c}10(20) \\
12(1-75) \\
19(2-98)\end{array}$ & $\begin{array}{c}13(62) \\
11(2-34) \\
11(1-34)\end{array}$ \\
\hline $\begin{array}{l}\text { Eventos adversos } \\
\text { Ansiedad } \\
\text { Inestabilidad } \\
\text { hemodinámica }^{\dagger} \\
\text { Intolerancia }^{\ddagger}\end{array}$ & $\begin{array}{l}1(2) \\
6(12) \\
1(2)\end{array}$ & $\begin{array}{l}2(9.5) \\
5(23.8)\end{array}$ \\
\hline
\end{tabular}

BUN: nitrógeno ureico sanguíneo; TOT: tubo orotraqueal; UCI: unidad de cuidados intensivos; VMNI: ventilación mecánica no invasiva.

*Las variables se presentan como promedio (mínimo-máximo) y valores absolutos (porcentaje), según la distribución de los datos.

'Definida como requerimiento de uso de vasopresor ante una presión arterial $\leq 90 / 60 \mathrm{mmHg}$ e hipoperfusión.

${ }^{\ddagger}$ Definida como ansiedad o incomodidad, barotrauma o vómito con el uso de VMNI que obligó a su retiro.

indica la intervención desde el ámbito prehospitalario o al inicio de la atención en urgencias ${ }^{3,28}$.

Los resultados de este estudio observacional sugieren un aumento no estadísticamente significativo de la mortalidad con el retardo en el inicio de la VMNI desde el ingreso al servicio de urgencias en los pacientes con EPC, desde un $5 \%$ por cada hora de retraso en el análisis de regresión logística hasta un 7\% en el análisis de variables instrumentales. El retraso del inicio de la intervención no se relacionó con mayor necesidad de intubaciones orotraqueales. El que los resultados descritos no permitan identificar una asociación, claramente son contraintuitivos, a partir de la experiencia con otras intervenciones en EPC que han demostrado éxito en modificar desenlaces cuanto más temprano se instauran.

Así como en el síndrome coronario agudo el concepto de la terapia guiada por tiempo es fundamental, parece que los tratamientos farmacológicos y no 
Tabla 3. Análisis multivariado para mortalidad hospitalaria según el tiempo de inicio de la ventilación mecánica no invasiva

\begin{tabular}{|l|c|c|}
\hline Variable & $\begin{array}{l}\text { Regresión logística } \\
\text { múltiple OR (IC95\%) }\end{array}$ & p \\
\hline $\begin{array}{l}\text { Tiempo de inicio de la VMNI } \\
\text { (variable continua) }\end{array}$ & $1.05(0.89-1.24)$ & 0.499 \\
\hline $\begin{array}{l}\text { Edad (variable continua) } \\
\text { Sexo femenino }\end{array}$ & $1.10(1.00-1.22)$ & 0.045 \\
\hline $\begin{array}{l}\text { Evolución aguda de la falla } \\
\text { cardíaca }\end{array}$ & $0.48(0.06-3.44)$ & 0.469 \\
\hline $\begin{array}{l}\text { Episodios previos de } \\
\text { descompensación }\end{array}$ & $1.74(0.06-47.41)$ & 0.743 \\
\hline $\begin{array}{l}\text { Antecedente de anemia } \\
\text { EPOC }\end{array}$ & $2.67(0.06-102.53)$ & 0.597 \\
\hline $\begin{array}{l}\text { Factor desencadenante } \\
\text { reconocido }\end{array}$ & $0.10(0.003-2.96)$ & 0.186 \\
\hline $\begin{array}{l}\text { Necesidad de intubación } \\
\text { posterior a la VMNI }\end{array}$ & $0.91(0.07-7.61)$ & 0.801 \\
\hline $\begin{array}{l}\text { Días en UCl (variable continua) } \\
\text { Días de hospitalización } \\
\text { (variable continua) }\end{array}$ & $1.60(1.09-2.35)$ & 0.749 \\
\hline $\begin{array}{l}\text { BUN (variable continua) } \\
\text { Hemoglobina (variable }\end{array}$ & $0.59(0.40-0.87)$ & 0.015 \\
\hline $\begin{array}{l}\text { continua) } \\
\text { (0.28-21.31) }\end{array}$ & 0.409 \\
\hline & $0.72(0.43-1.18)$ & 0.194 \\
\hline
\end{tabular}

BUN: nitrógeno ureico sanguíneo; EPOC: enfermedad pulmonar obstructiva crónica; IC95\%: intervalo de confianza del 95\%; OR: odds ratio; UCI: unidad de cuidados intensivos; VMNI: ventilación mecánica no invasiva.

farmacológicos de la falla cardíaca aguda van en la misma dirección ${ }^{28}$, y de ahí la importancia de evitar los retrasos en su instauración por sus efectos en la mortalidad y otros desenlaces adversos. Un estudio que exploró el impacto del tiempo de las intervenciones médicas en el EPC fue el estudio del tiempo al inicio de furosemida en 2017, en el que se comparó la mortalidad intrahospitalaria en pacientes con falla cardíaca aguda que recibieron terapia diurética de manera temprana, definida como menor de 60 minutos desde el ingreso, frente a los que la recibieron en forma tardía. Se encontró una reducción de la mortalidad en el grupo de terapia temprana: $2.3 \%$ frente a $6 \%$ (OR: 0.39 ; IC95\%: 0.20-0.76) $)^{30}$.

El estudio de Plaisance et al. ${ }^{31}$ abordó los efectos de la CPAP como primera línea de tratamiento prehospitalario del EPC en un grupo de 124 pacientes, con una mediana de edad de 77 años y con miocardiopatía y arritmia como principales etiologías, recibiendo manejo médico con bumetanida, nitroglicerina y nicardipino. Encontraron que los expuestos en la intervención de los primeros 15 minutos de atención (grupo de terapia temprana) presentaron como desenlaces primarios menos disnea y menos acidosis, y alcanzaron mejores índices de oxigenación que aquellos que solo recibieron tratamiento farmacológico (grupo tardío) en el mismo tiempo, seguido por CPAP 15 minutos después. Como desenlaces secundarios, la mortalidad en el grupo de terapia temprana fue menor (OR: 0.22; IC95\%: 0.04-1.0), al igual que la necesidad de intubación orotraqueal (OR: 0.30; IC95\%: 0.09-0.89) ${ }^{31}$. En nuestro estudio se analizaron, con una muestra menor, pacientes más jóvenes y con cardiopatía isquémica e hipertensiva como principales desencadenantes de EPC, recibiendo manejo médico con furosemida y nitroglicerina, y como desenlace primario se quiso estudiar la posible correlación entre la mortalidad con el retraso de la intervención y la reducción en la necesidad de intubación. Los tiempos requeridos para el inicio de la VMNI fueron distintos: mientras que para Plaisance et al. ${ }^{31}$ se trataba de 15 minutos, en nuestro estudio se analizó solo el tiempo dentro de las primeras 24 horas de atención intrahospitalaria. Es posible que los resultados del primero confirmen la hipótesis de que la CPAP iniciada tempranamente en el EPC (incluso antes del ingreso a urgencias) genere una reducción en la mortalidad, pero podría ser que las primeras 24 horas del cuadro sean un período muy amplio en el que ese beneficio pudiera perderse en algún momento, esto considerando que la mayoría de los estudios sobre el tema solo evaluaron este lapso de tiempo específico ${ }^{10,29}$.También consideramos que el beneficio pudiera limitarse a subtipos de pacientes según la edad, la fracción de eyección (este parámetro no fue estudiado por Plaisance et al. ${ }^{31}$ ) y las causas del EPC, lo que pudiera explicar por qué no logramos encontrar resultados contundentes entre nuestra población.

Además, la falta de significado estadístico en la asociación del retraso en el inicio de la VMNI con el desenlace primario podría ser explicable por otras razones, entre ellas el tamaño de la muestra, la naturaleza retrospectiva del estudio, la selección de los pacientes basada en el código CIE-10 e incluso la falta de exploración de algunas variables que pueden modificar la decisión del clínico para iniciar más o menos temprano la intervención.

Por último, se identificó una gran variabilidad en la forma en que se administra el tratamiento farmacológico en el EPC, tanto en las dosis de diuréticos como en el uso de venodilatador, lo cual pone en evidencia la heterogeneidad en la aplicación de las guías de 
práctica clínica por parte de los tratantes y algunas diferencias en las cointervenciones de las fuentes reseñadas anteriormente; este elemento también podría impactar en los resultados encontrados.

\section{Limitaciones}

Este estudio tiene algunas limitaciones que son inherentes a los estudios de tipo observacional y retrospectivo. Entre estas se encuentran la falta de datos en varias de las historias, la asignación errónea del diagnóstico de EPC, la falta de claridad en las causas precipitantes de EPC y, como aspecto más relevante en este estudio en particular, que los datos sobre el inicio de la VMNI fueron tomados a partir de la hora en que se realizó el registro en la historia clínica. Para tratar de evitar al máximo que esto afectara los resultados finales del estudio, se realizó una recolección estricta de la información a partir de la historia clínica del paciente y no solo de la impresión diagnóstica al momento del ingreso; de esta forma, fue posible evitar incluir en el análisis pacientes con otros diagnósticos y conservar la rigurosidad en cuanto a los criterios de inclusión y exclusión. Por otra parte, en la evaluación de intervenciones en estudios observacionales es posible que se presente un «sesgo de confusión por indicación» ${ }^{32}$, que señala que los pacientes con peor pronóstico son los de mayor probabilidad de recibir la terapia en estudio; este sesgo se intentó minimizar por medio del análisis de variables instrumentales, mientras que el análisis de regresión logística buscó controlar al máximo las variables de confusión, pero no podemos descartar la confusión residual ni el subajuste por problemas del tamaño de muestra. Algunas variables, como la frecuencia respiratoria, la saturación de oxígeno o los gases arteriales desde el ingreso, pueden influir en el tiempo de inicio de la VMNI, y otra limitación de este estudio fue que no se obtuvieron estos datos.

El control de sesgos estuvo principalmente ligado a la revisión estricta de la información de las historias clínicas por parte de los investigadores. Para el control de las variables de confusión se llevó a cabo una revisión de la literatura con el fin de cruzar y verificar información sobre el área de estudio; además, se realizó una regresión logística múltiple para ajustar las variables respectivas ${ }^{33}$.

\section{Conclusiones}

En este estudio de cohorte observacional retrospectiva, los resultados sugieren un aumento de la mortalidad, no significativo estadísticamente, entre el $5 \%$ y el $7 \%$ por cada hora de retraso en el inicio de la intervención; sin embargo, consideramos que hace falta explorar este panorama y así generar evidencia concluyente.

\section{Agradecimientos}

Los autores agradecen a la doctora Clara Inés Saldarriaga, internista cardióloga, por su asesoría y aportes en la revisión del manuscrito.

\section{Financiamiento}

El estudio fue financiado en su totalidad por los autores.

\section{Conflicto de intereses}

Los autores declaran no tener conflictos de intereses.

\section{Bibliografía}

1. Marteles MS, Urrutia A. Formas de presentación de la insuficiencia cardíaca aguda: edema agudo de pulmón y shock cardiogénico. Med Clin (Barc). 2014;142:14-9.

2. Ponikowski $\mathrm{P}$, Voors AA, Anker SD, Bueno H, Cleland JGF, Coats AJS, et al. Guía ESC 2016 sobre el diagnóstico y tratamiento de la insuficiencia cardíaca aguda y crónica. Rev Esp Cardiol. 2016;69:1167.e1-85.

3. National Institute for Health and Care Excellence (NICE). Acute Heart Failure: diagnosis and management. UK: NICE: 2014. Disponible en: https://www.nice.org.uk/guidance/cg187/resources/acute-heart-failure-diagnosis-and-management-pdf-35109817738693

4. Masip J, Peacock WF, Price S, Cullen L, Martín-Sánchez FJ, Seferovic $P$, et al. Indications and practical approach to non-invasive ventilation in acute heart failure. Eur Heart J. 2018;39:17-25.

5. Ho KM, Wong K. A comparison of continuous and bi-level positive airway pressure non-invasive ventilation in patients with acute cardiogenic pulmonary oedema: a meta-analysis. Crit Care. 2006;10:R49.

6. Collins SP, Mielniczuk LM, Whittingham HA, Boseley ME, Schramm DR, Storrow $\mathrm{AB}$. The use of noninvasive ventilation in emergency department patients with acute cardiogenic pulmonary edema: a systematic review. Ann Emerg Med. 2006;48:260-9, 269.e1-4.

7. Gray A, Goodacre S, Newby DE, Masson M, Sampson F, Nicholl J, et al. Noninvasive ventilation in acute cardiogenic pulmonary edema. $\mathrm{N}$ Engl J Med. 2008;359:142-51.

8. Weng $\mathrm{CL}$, Zhao YT, Liu QH, Fu CJ, Sun F, Ma YL, et al. Noninvasive ventilation in acute cardiogenic pulmonary edema. Ann Intern Med. 2010;152:590-600.

9. Vital FMR, Ladeira MT, Atallah AN. Non-invasive positive pressure ventilation (CPAP or bilevel NPPV) for cardiogenic pulmonary oedema. Cochrane Database Syst Rev. 2013;(5):CD005351.

10. Park M, Sangean MC, Volpe M de S, Feltrim MIZ, Nozawa E, Leite PF, et al. Randomized, prospective trial of oxygen, continuous positive airway pressure, and bilevel positive airway pressure by face mask in acute cardiogenic pulmonary edema. Crit Care Med. 2004;32:2407-15.

11. Mehta S, Al-Hashim AH, Keenan SP. Noninvasive ventilation in patients with acute cardiogenic pulmonary edema. Respir Care. 2009;54:186-97.

12. Rochwerg B, Brochard L, Elliott MW, Hess D, Hill NS, Nava S, et al. Official ERS/ATS clinical practice guidelines: noninvasive ventilation for acute respiratory failure. Eur Respir J. 2017;50:1602426.

13. Yancy CW, Jessup M, Bozkurt B, Butler J, Casey DE, Colvin MM, et al. 2017 ACC/AHA/HFSA focused update of the 2013 ACCF/AHA Guideline for the management of heart failure. J Am Coll Cardiol. 2017;70:776-803.

14. MinSalud-Colombia. Guía de práctica clínica para la prevención, diagnóstico, tratamiento y rehabilitación de la falla cardíaca en población mayor de 18 años clasificación B, C y D. Colombia: MinSalud; 2015.

15. Cleland JGF, Yassin AS, Khadjooi K. Acute heart failure: focusing on acute cardiogenic pulmonary oedema. Clin Med. 2010;10:59-64. 
16. Assaad S, Kratzert WB, Shelley B, Friedman MB, Perrino A. Assessment of pulmonary edema: principles and practice. J Cardiothorac Vasc Anesth. $2018 ; 32: 901-14$.

17. Hospital San Vicente Fundación, nuestras entidades: Hospital Medellín. (Consultado 10-08-2020.) Disponible en: https://www.sanvicentefundacion.com/nuestras-entidades/hospital-medellin.

18. Celentano D, Szklo M. Más sobre inferencias causales: sesgos, confusión e interacción. En: Gordis L. Epidemiología. Madrid: Elsevier; 2014. p. 262-78.20

19. Tarvasmäki T, Harjola V-P, Nieminen MS, Siirilä-Waris K, Tolonen J, Tolppanen $\mathrm{H}$, et al. Acute heart failure with and without concomitant acute coronary syndromes: patient characteristics, management, and survival. J Card Fail. 2014;20:723-30.

20. Conte P Le, Coutant V, N'Guyen JM, Baron D, Touzé MD, Potel G. Prognostic factors in acute cardiogenic pulmonary edema. Am J Emerg Med. 1999;17:329-32.

21. Figueras J, Bañeras J, Peña-Gil C, Barrabés JA, Palomares JR Dorado DG. Hospital and 4-year mortality predictors in patients with acute pulmonary edema with and without coronary artery disease. J Am Heart Assoc. 2016;5:e002581.

22. Rohde LE, Goldraich L, Polanczyk CA, Borges AP, Biolo A, Rabelo E, et al. A simple clinically based predictive rule for heart failure in-hospital mortality. J Card Fail. 2006;12:587-93.

23. Aronson D, Mittleman MA, Burger AJ. Elevated blood urea nitrogen level as a predictor of mortality in patients admitted for decompensated heart failure. Am J Med. 2004;116:466-73.

24. Yancy CW, Lopatin M, Stevenson LW, De Marco T, Fonarow GC. Clinical presentation, management, and in-hospital outcomes of patients admitted with acute decompensated heart failure with preserved systolic function: a report from the Acute Decompensated Heart Failure National Registry (ADHERE) database. J Am Coll Cardiol. 2006;47:76-84.
25. Greenland S. An introduction to instrumental variables for epidemiologists. Int J Epidemiol. 2000;29:722-9.

26. Muñoz M, Giraldo D, Jaimes F. Ronda clínica y epidemiológica. Uso de variables instrumentales en investigación médica. latreia. 2013;26:371-9

27. Baum CF, Schaffer ME, Stillman S. Instrumental variables and GMM: estimation and testing. Stata $\mathrm{J}$ Promot Commun Stat Stata. 2003:3:1-31.

28. Mebazaa A, Yilmaz MB, Levy P, Ponikowski P, Peacock WF, Laribi S, et al. Recommendations on pre-hospital and early hospital management of acute heart failure: a consensus paper from the Heart Failure Association of the European Society of Cardiology, the European Society of Emergency Medicine and the Society of Academic Emergency Medicine. Eur J Heart Fail. 2015;17:544-8.

29. Berbenetz N, Wang Y, Brown J, Godfrey C, Ahmad M, Vital FM, et al. Non-invasive positive pressure ventilation (CPAP or bilevel NPPV) for cardiogenic pulmonary oedema. Cochrane Database Syst Rev. 2019;(4):CD005351.

30. Matsue Y, Damman K, Voors AA, Kagiyama N, Yamaguchi T, Kuroda S, et al. Time-to-furosemide treatment and mortality in patients hospitalized with acute heart failure. J Am Coll Cardiol. 2017;69:3042-51.

31. Plaisance P, Pirracchio R, Berton C, Vicaut E, Payen D. A randomized study of out-of-hospital continuous positive airway pressure for acute cardiogenic pulmonary oedema: physiological and clinical effects. Eur Heart J. 2007;28:2895-901.

32. Kyriacou DN, Lewis RJ. Confounding by indication in clinical research. $\mathrm{J}$ Am Med Assoc. 2016;316:1818-9

33. Londoño Fernández JF. Fuentes de error en investigación epidemiológica: el azar y los sesgos. En: Londoño Fernández JF. Metodología de la investigación epidemiológica. Bogotá: El Manual Moderno; 2017. p. 101-19. 